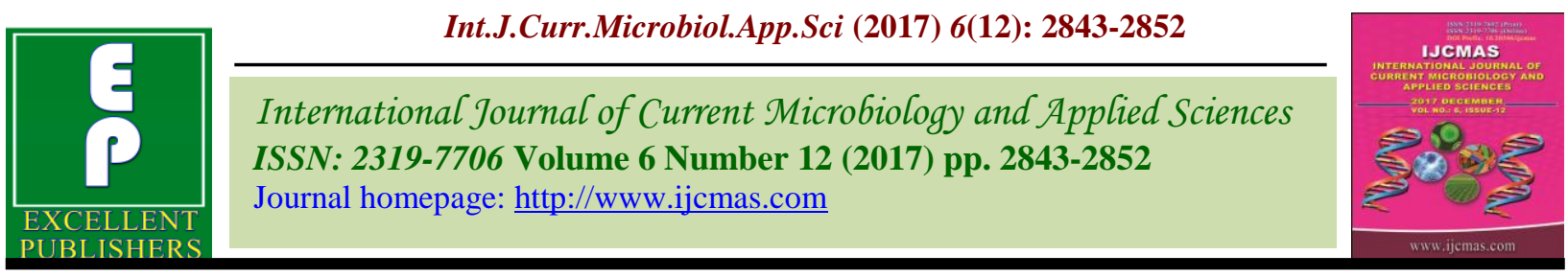

Original Research Article

https://doi.org/10.20546/ijcmas.2017.612.331

\title{
Exploitation of Heterosis for Yield and its Components in Castor (Ricinus communis L.)
}

\author{
Sumit G. Gajbhe, S.B. Sakhare and S.R. Rathi* \\ Department of Agricultural Botany, Dr. Panjabrao Deshmukh Krishi Vidyapeeth, \\ Akola Maharashtra, India \\ *Corresponding author
}

A B S T R A C T

Keywords

Average heterosis,

Heterosis,

Heterobeltiosis,

Standard heterosis, Castor.

\section{Article Info}

Accepted:

19 October 2017

Available Online:

10 December 2017
An experiment was conducted to study the nature and magnitude of heterosis for seed yield and its fifteen yield attributing components along with oil content. Four females and five males were crossed in a line $\mathrm{x}$ tester fashion to develop $20 \mathrm{~F}_{1}$ hybrids. The resulted $20 \mathrm{~F}_{1}$ hybrids and their nine parents along with two standard checks (AKC-1 and 48-1) were evaluated in randomized block design with three replications at the field of AICRP for Dryland Agriculture, Dr. PDKV, Akola during kharif 2015-16. The genotypes, parents and crosses revealed high degree of differences for all the characters under study. The highest useful heterosis of 39.66 per cent over the check variety AKC-1 and 53.05 per cent over the check variety 48-1 for seed yield was observed indicating the scope of its exploitation further in the breeding programme of this crop. The cross AKD1 X 561-2 has been found to be promising and can be utilized after confirming its potential on large scale.

\section{Introduction}

Castor (Ricinus communis L.) with $2 \mathrm{n}=20$, belongs to the family Euphorbiaceae and it is indigenous to eastern Africa and most probably originated in Ethiopia. The genus Ricinus is monotypic and $R$. communis is the only species with the most polymorphic forms known. In castor, its monoecious nature favours cross pollination, and it is up to the extent of 50 per cent. It is grown in tropical, sub-tropical and temperate regions of the world. It is cultivated in about 30 countries on commercial scale. India is the world's principal producer of castor and ranks first both in area and production. Castor productivity in India is more than world average and it ranks first among the major castor producing countries viz., India, China, Brazil and Thailand. Castor seed contains 47 to 55 per cent oil. Due to its unique chemical and physical properties, the oil from castor seed is used as an important industrial nonedible oil seed crop. The phenomenon of heterosis has proved to be the most important genetic tool in enhancing the yield of self as well as cross pollinated crop species in general and castor in particular. With the availability of cent per cent pistillate lines in castor, exploitation of heterosis or hybrid vigour on commercial scale has become commercially feasible and economical the 
exploitation of heterosis on commercial scale in castor is regarded as one of the major breakthrough in the improvement of productivity of castor. In order to achieve high-yielding cross combinations, it is essential to evaluate available promising diverse lines and their hybrid combinations for yield and its attributes.

\section{Materials and Methods}

The experimental material for the present investigation were generated by crossing four lines namely AKC-1, 48-1, Aruna and AKD-1 and five testers namely 561-2, 592-2, 554-1, 636-5 and 2-1-12 during kharif 2014-15 to obtain 20 F1's. These 20 F1's and their parents including the checks AKC-1 and 48-1 were evaluated during kharif 2015-16. Nine parents, their hybrids and two checks were raised in a randomized complete block design in two replications. Recommended agronomic practices were followed to grow a healthy crop. Observations were recorded on days to $50 \%$ flowering and days to maturity of primary spikes, number of nodes up to primary spikes, plant height, effective length of primary spikes, effective length of secondary spikes, effective length of tertiary spikes, number of secondary spikes, number of tertiary spikes, total number of spikes, number of capsules on primary spikes, number of capsules on secondary spikes, number of capsules on tertiary spikes, total number of capsules, 100 seed weight, seed yield and oil content.

The data recorded on the material generated as per Line $\times$ Tester model of Kempthorne (1957) were subjected to analysis of variance as per the method suggested by Panse and Sukhatme Heterosis expressed as per cent increase or decrease in the mean value of $\mathrm{F} 1$ hybrid over mid parent i.e., average heterosis. Heterobeltiosis was estimated as per the procedure given by Fonseca and Patterson
(1968). Standard heterosis referred as the superiority of F1 over standard hybrid AKC1and 48-1 and it was estimated as per the formula given by Meredith and Bridge (1972) for various characters under study.

\section{Results and Discussion}

The analysis of variance was performed to test the significant differences among genotypes, parents, hybrids and parents vs. hybrids for all the sixteen characters studied and are presented in Table 1. The results revealed that mean squares due to genotypes were highly significant for all the characters except plant height. The mean squares due to genotypes were further partitioned into mean squares due to parents, hybrids and parents vs. hybrids. The analysis of variance revealed that mean squares due to hybrids highly significantly for all the characters except oil content, but mean squares due to parents were significant for all the traits, except plant height and oil content. This indicated the existence of considerable genetic variability among the parents and hybrids for all the characters under study. The mean squares due to parents vs. hybrids were also significant for all the characters except plant height, total number of spikes and oil content, which indicated that the performance of parents as a group was different than that of crosses as a group, thereby supporting the presence of mean heterosis for all the traits studied. The results are in accordance to those reported by Ramana et al., (2005) and Sapovadiya et al., (2015) in castor.

Considering the per se performance of hybrids, 5 crosses significantly out yielded the standard check 48-1 (57.67 g) whereas only four crosses showed superiority over AKC-1 $(63.20 \mathrm{~g})$ for seed yield per plant. The cross AKD1 x 561-2 (88.26g) was found to be superior cross in respect of seed yield followed by the cross Aruna x 561-2 (75.25 
g). Their superiority seemes to have been resulted due to yield contributing characters particularly effective length of primary spikes, effective length of secondary spikes, number of capsules on primary spikes and total number of capsules per plant. The cross AKD1 x 561-2 showed significant superiority for the traits viz., effective length of primary spikes $(39.67 \mathrm{~cm})$, effective length of secondary spikes (19.45), number of capsules on primary spikes (76.23), number of capsules on secondary spikes (24.00), number of capsules on tertiary spikes (2.93) and total number of capsules per plant (108.88) and also seed yield per plant (88.26 g). The cross Aruna x 2-1-12 exhibited maximum mean performance for the characters viz., number of tertiary spikes (6.83), number of secondary spikes (4.33), effective length of tertiary spike $(2.82 \mathrm{~cm})$ and total number of spikes $(6.83)$.

The results of heterotic effects over mid parent $(\mathrm{RH})$, better parent $(\mathrm{HB})$ and over checks $(\mathrm{SH})$ for various characters have been presented in Table 2, 3 and $4 \mathrm{a}$ and $\mathrm{b}$ respectively which revealed that the magnitude and extent of various heterotic effects varied with cross combinations and characters, which indicated that parents differed for contributing increasing and decreasing genes causing dominance effect. Thus, a good number of crosses had significant heterosis over the mid parent, better parent and standard checks AKC-1 and 48-1 for various traits in desired direction. The highest average heterosis (Table 2) in desirable direction was recorded in the cross AKD1 X 561-1 for the characters viz., effective length of primary spike (97.84\%), effective length of secondary spikes (170.08\%), effective length of tertiary spikes (287.15\%), number of tertiary spikes (130.72), total number of spikes (117.14), number of capsules on primary spikes (115\%), number of capsules on secondary spikes $(126.83 \%)$, number of capsules on tertiary spikes (314.60\%), total number of capsules per plant $(123.57 \%)$ and seed yield per plant (118.05\%). The cross Aruna x 592-2 exhibited maximum average heterosis for number of secondary spikes (67.32\%). Different crosses exhibited highest average heterosis for different characters viz., The cross Aruna X 2-1-12 exhibited negative desirable highest heterosis for days to $50 \%$ flowering (-21.76\%), the cross 48-1 x 561-2 ($14.54 \%$ ) for days to maturity of primary spike, the cross AKC1 x 561-2 for number of nodes to primary spike $(-10.82 \%)$, the cross 48-1 x 554-1 for 100 seed weight $(6.26 \%)$ and AKC1 x 2-1-12 for oil content (7.54\%).

Many crosses exhibited maximum heterosis over the better parent (Table 3) in desirable direction for yield and its component traits. The cross AKD1 X 561-2 showed highest heterobeltiosis for maximum number of characters viz., effective length of primary spikes $(71.72 \%)$, effective length of secondary spikes (124.77\%), number of secondary spikes (43.29\%), effective length of tertiary spikes $(287.15 \%)$, number of tertiary spikes $(130.72 \%)$, total number of spikes (95.30\%), number of capsules on primary spike $(71.61 \%)$, number of capsules on secondary spike (106.39), number of capsule per plant $(97.34 \%)$ and seed yield (114.60\%). The cross AKC1 X 561-2 exhibited desirable negative heterosis over better parent for the characters, number nodes upto primary spikes $(-8.58 \%)$. The cross Aruna $X$ 2-1-12 exhibited highest negative heterosis for days to $50 \%$ flowering $(-21.76$ days) and the cross 48-1 x 554-1 (-5.53\%) for days to maturity of primary spikes. It was observed that cross showing high heterobeltosis for seed yield per plant in general also manifested heterotic effects for its contributing characters like effective length of tertiary spikes, number of tertiary spikes and number of capsules on secondary spikes. 
Table.1 Analysis of variance for various characters

\begin{tabular}{|c|c|c|c|c|c|c|c|c|c|c|}
\hline \multirow[t]{2}{*}{ Sources of variation } & \multirow[t]{2}{*}{ DF } & \multicolumn{9}{|c|}{ Mean sum of squares } \\
\hline & & $\begin{array}{l}\text { Days to } 50 \% \\
\text { flowering of } \\
\text { primary spikes }\end{array}$ & $\begin{array}{l}\text { Days to } \\
\text { maturity of } \\
\text { primary } \\
\text { spikes }\end{array}$ & $\begin{array}{l}\text { Number of } \\
\text { nodes up } \\
\text { to primary } \\
\text { spikes }\end{array}$ & $\begin{array}{l}\text { Plant height } \\
\text { (cm) }\end{array}$ & $\begin{array}{l}\text { Effective } \\
\text { length of } \\
\text { primary } \\
\text { spikes }\end{array}$ & $\begin{array}{l}\text { Effective } \\
\text { length of } \\
\text { secondary } \\
\text { spikes }\end{array}$ & \begin{tabular}{|l} 
Effective \\
length of \\
tertiary \\
spikes
\end{tabular} & $\begin{array}{l}\text { Number of } \\
\text { secondary } \\
\text { spikes }\end{array}$ & $\begin{array}{l}\text { Number of } \\
\text { tertiary } \\
\text { spikes }\end{array}$ \\
\hline Replications & 2 & $1.27 * *$ & 10.08 & 3.35 & 51.65 & 0.90 & 1.52 & 0.003 & 0.03 & 0.01 \\
\hline Treatments & 28 & $117.50 * *$ & $207.83 * *$ & $14.08 * *$ & 1895.83 & $134.87 * *$ & $32.73 * *$ & $2.649 * *$ & $3.08 * *$ & $0.53 * *$ \\
\hline Parents & 8 & $155.78 * *$ & $557.83 * *$ & $25.15 * *$ & 2778.81 & $166.60 * *$ & $27.65 * *$ & $2.830^{* * *}$ & $3.35 * *$ & $0.79 * *$ \\
\hline Crosses & 19 & $93.27 * *$ & $43.82 * *$ & $6.97 * *$ & $705.10 * *$ & $128.59 * *$ & $33.37 * *$ & $2.587 * *$ & $3.09 * *$ & $0.73^{* *}$ \\
\hline Parents Vs Crosses & 1 & $271.77 * *$ & $524.15^{* *}$ & $60.52 * *$ & 17455.95 & $0.34 * *$ & $61.32 * *$ & $2.384 * *$ & $0.76^{* *}$ & $0.41 * *$ \\
\hline Error & 56 & 2.22 & 7.78 & 2.77 & 30.71 & 3.85 & 0.50 & 0.00278 & 0.06 & 0.01 \\
\hline
\end{tabular}

Table.1 Contd...

\begin{tabular}{|c|c|c|c|c|c|c|c|c|c|}
\hline \multirow[t]{2}{*}{ Sources of variation } & \multirow[t]{2}{*}{ DF } & \multicolumn{8}{|c|}{ Mean sum of squares } \\
\hline & & $\begin{array}{l}\text { Total number } \\
\text { of spikes }\end{array}$ & \begin{tabular}{|l|} 
Number of \\
capsules on \\
primary spikes
\end{tabular} & $\begin{array}{l}\text { Number of } \\
\text { capsules on } \\
\text { secondary } \\
\text { spikes } \\
\end{array}$ & $\begin{array}{l}\text { Number of } \\
\text { capsules on } \\
\text { tertiary spikes }\end{array}$ & $\begin{array}{l}\text { Total number } \\
\text { of capsule } \\
\text { plant }^{-1}\end{array}$ & $\begin{array}{l}100 \text { seed } \\
\text { weight }(\mathrm{g})\end{array}$ & \begin{tabular}{|l|} 
Seed yield \\
plant $^{-1}$
\end{tabular} & Oil content \\
\hline Replications & 2 & 0.28 & 0.43 & 2.33 & 0.007 & 3.77 & 0.01 & 0.83 & 2.05 \\
\hline Treatments & 28 & $9.50 * *$ & $655.25 * *$ & $40.28 * *$ & $2.863 * *$ & $985.72 * *$ & $11.49^{* *}$ & $653.67 * *$ & $2.33^{*}$ \\
\hline Parents & 8 & $14.37 * *$ & $502.10 * *$ & $59.33 * *$ & $3.456 * *$ & $734.78 *$ & $19.71 * *$ & $478.49 * *$ & 2.44 \\
\hline Crosses & 19 & $7.42 * *$ & $681.95 * *$ & $33.50 * *$ & $1.718 * *$ & $1158.33 * *$ & $7.68 * *$ & $760.22 * *$ & 2.35 \\
\hline Parents Vs Crosses & 1 & 10.01 & $1373.23 * *$ & $4.51 * *$ & $2.764 * *$ & $283.81 * *$ & $17.68 * *$ & $30.80 * *$ & 1.10 \\
\hline Error & 56 & 0.75 & 1.12 & 3.60 & 0.005 & 3.00 & 0.26 & 1.96 & 1.39 \\
\hline
\end{tabular}

Note: * Significant at 5\% level of significance, $* *$ Significant at $1 \%$ level of significance.

Table.2 Average heterosis (\%) for various characters

\begin{tabular}{|c|c|c|c|c|c|c|c|c|c|}
\hline $\begin{array}{l}\text { Sr. } \\
\text { No. }\end{array}$ & Crosses & $\begin{array}{l}\text { Days to } 50 \% \\
\text { flowering of } \\
\text { primary } \\
\text { spikes }\end{array}$ & $\begin{array}{l}\text { Days to } \\
\text { maturity of } \\
\text { primary } \\
\text { spikes }\end{array}$ & $\begin{array}{l}\text { Number of } \\
\text { nodes up } \\
\text { to primary } \\
\text { spikes } \\
\end{array}$ & $\begin{array}{l}\text { Effective } \\
\text { length of } \\
\text { primary } \\
\text { spikes }\end{array}$ & $\begin{array}{l}\text { Effective } \\
\text { length of } \\
\text { secondary } \\
\text { spikes }\end{array}$ & $\begin{array}{l}\text { Effective } \\
\text { length of } \\
\text { tertiary } \\
\text { spikes }\end{array}$ & $\begin{array}{l}\text { Number of } \\
\text { secondary } \\
\text { spikes }\end{array}$ & $\begin{array}{l}\text { Number of } \\
\text { tertiary spike }\end{array}$ \\
\hline & & 1 & 2 & 3 & 4 & 5 & 6 & 7 & 8 \\
\hline 1 & AKC-1 X 561-2 & 0.23 & $-12.59 * *$ & $-10.82 *$ & $15.83 * *$ & $36.52 * *$ & $57.52 * *$ & $-20.39 * *$ & $24.69 * *$ \\
\hline 2 & AKC-1 X 592-2 & $3.52 *$ & $-3.39 *$ & 6.62 & $-40.54 * *$ & 6.92 & $74.27 * *$ & -2.97 & 7.85 \\
\hline 3 & AKC-1 X554-1 & $7.34 * *$ & $-2.73 *$ & 4.02 & $-41.60 * *$ & $-29.09 * *$ & $-70.03 * *$ & -4.21 & $-55.01 * *$ \\
\hline 4 & AKC-1 X636-5 & $-10.77 * *$ & -1.91 & 8.77 & -6.03 & 3.99 & $-52.51 * *$ & -11.56 & $-40.79 * *$ \\
\hline 5 & AKC-1 X 2-1-12 & -1.18 & $3.14 *$ & 10.16 & $19.06 * *$ & $10.72 *$ & $-71.64 * *$ & $-11.88 * *$ & $-60.33 * *$ \\
\hline 6 & AKD1 x561-2 & -2.19 & $-13.25 * *$ & 9.45 & $97.84 * *$ & $170.08 * *$ & $287.15 * *$ & 67.17 ** & $130.72 * *$ \\
\hline 7 & AKD1x592-2 & -0.92 & 0.34 & 12.55 & $-42.99 * *$ & $25.93 * *$ & 0 & 6.91 & 0 \\
\hline 8 & AKD1X 554-1 & $14.67 * *$ & 1.93 & 43.86 ** & $19.70 * *$ & -9.47 & $-55.18 * *$ & $-53.85 * *$ & $-34.81 * *$ \\
\hline 9 & AKD1X 636-5 & -0.23 & -0.67 & $13.89 *$ & $23.85 * *$ & $-25.17 * *$ & 0 & $-47.83 * *$ & 0 \\
\hline 10 & AKD1X 2-1-12 & $-18.52 * *$ & 0.93 & 31.97 ** & $59.84 * *$ & $60.27 * *$ & $36.83 * *$ & $-23.08 * *$ & 4.31 \\
\hline 11 & $48-1 \times 561-2$ & $-11.84 * *$ & $-14.54 * *$ & 8.4 & $53.98 * *$ & $103.31 * *$ & $-57.81 * *$ & $40.83 * *$ & $-41.63 * *$ \\
\hline 12 & $48-1 \times 592-2$ & $-13.99 * *$ & $4.20 * *$ & 4.94 & -6.39 & $21.50 * *$ & $71.46 * *$ & $14.63 *$ & $34.67 * *$ \\
\hline 13 & $48-1$ X 554-1 & $4.29 * *$ & $-5.35 * *$ & $31.55 * *$ & -8.4 & $17.48 * *$ & $-72.23 * *$ & 2.74 & $-55.50 * *$ \\
\hline 14 & $48-1$ X 636-5 & $-19.17 * *$ & -2.31 & 5.38 & -8.36 & $-26.70 * *$ & $-57.81 * *$ & $-57.66 * *$ & $-41.63 * *$ \\
\hline 15 & $48-1 \times 2-1-12$ & $-8.89 * *$ & -1.71 & $18.29 * *$ & 6.41 & $-13.71 *$ & $-73.61 * *$ & $-72.94 * *$ & $-60.71 * *$ \\
\hline 16 & Aruna x561-2 & $-5.94 * *$ & $-10.58 * *$ & 0.58 & $10.78 *$ & $91.58 * *$ & $-57.17 * *$ & $-40.00 * *$ & -10.87 \\
\hline 17 & Aruna x 592-2 & $-10.63 * *$ & -1.23 & $15.34 *$ & $-46.27 * *$ & $-14.41 * *$ & $44.17 * *$ & $67.32 * *$ & $62.33 * *$ \\
\hline 18 & Aruna x554-1 & $14.93 * *$ & -1.01 & $24.81 * *$ & $-16.66 * *$ & -6.32 & $-71.95 * *$ & $57.64 * *$ & $-39.61 * *$ \\
\hline 19 & Aruna x636-5 & $-10.39 * *$ & $2.89 *$ & 2.74 & $-22.44 * *$ & $-30.78 * *$ & $-57.17 * *$ & $-36.84 * *$ & -10.87 \\
\hline \multirow[t]{4}{*}{20} & Aruna x 2-1-12 & $-21.76 * *$ & 0.92 & $16.59 *$ & $25.11 * *$ & $57.67 * *$ & $6.50 * *$ & 48.57 ** & 1.82 \\
\hline & $\mathrm{SE}(\mathrm{m}) \pm$ & 1.06 & 1.97 & 1.18 & 1.39 & 0.5 & 0.0373 & 0.17 & 0.0642 \\
\hline & CD AT $5 \%$ & 2.14 & 3.99 & 2.38 & 2.81 & 1.01 & 0.0755 & 0.34 & 0.13 \\
\hline & CD AT $1 \%$ & 2.86 & 5.35 & 3.19 & 3.76 & 1.36 & 0.1011 & 0.45 & 0.1742 \\
\hline
\end{tabular}

Note: * - Significant at $5 \%$ level of significance $* *$ - Significant at $1 \%$ level of significance 
Table. 2 Contd....

\begin{tabular}{|c|c|c|c|c|c|c|c|c|c|}
\hline $\begin{array}{l}\text { Sr. } \\
\text { No. }\end{array}$ & Crosses & $\begin{array}{c}\text { Total } \\
\text { Number of } \\
\text { spikes }\end{array}$ & $\begin{array}{c}\text { Number of } \\
\text { capsules on } \\
\text { primary } \\
\text { spikes } \\
\end{array}$ & $\begin{array}{c}\text { Number of } \\
\text { capsules on } \\
\text { secondary } \\
\text { spikes }\end{array}$ & $\begin{array}{c}\text { Number of } \\
\text { capsules on } \\
\text { tertiary } \\
\text { spikes } \\
\end{array}$ & $\begin{array}{c}\text { Total number } \\
\text { of capsule per } \\
\text { plant }\end{array}$ & $\begin{array}{c}100 \text { seed } \\
\text { weight }\end{array}$ & $\begin{array}{c}\text { Seed yield } \\
\text { per plant }\end{array}$ & Oil content \\
\hline & & 9 & 10 & 11 & 12 & 13 & 14 & 15 & 16 \\
\hline 1 & AKC-1 X 561-2 & 0 & $36.72 * *$ & $7.78 *$ & $49.93 * *$ & $31.44 * *$ & -1.51 & $27.60 * *$ & -0.11 \\
\hline 2 & AKC-1 X 592-2 & -1.69 & $34.50 * *$ & $25.02 * *$ & $61.97 * *$ & $-26.27 * *$ & $-11.00 * *$ & $-36.19 * *$ & -0.39 \\
\hline 3 & AKC-1 X554-1 & $-36.05 * *$ & $-4.16 *$ & $-31.21 * *$ & $-73.28 * *$ & $-46.88 * *$ & $3.11 *$ & $-45.54 * *$ & -2.26 \\
\hline 4 & AKC-1 X636-5 & $-30.65 * *$ & $13.74 * *$ & $-8.90 * *$ & $-56.69 * *$ & $-28.81 * *$ & $-4.45 * *$ & $-33.00 * *$ & -3.24 \\
\hline 5 & AKC-1 X 2-1-12 & $-41.32 * *$ & 18.75 ** & 5.3 & $-74.40 * *$ & $-7.57 * *$ & -0.81 & $-9.22 * *$ & $7.54 * *$ \\
\hline 6 & AKD1 x561-2 & $117.00 * *$ & $115.99 * *$ & 126.83 ** & $314.60 * *$ & $123.57 * *$ & -2.53 & $118.05 * *$ & $-4.12 *$ \\
\hline 7 & AKD1x592-2 & 4.63 & $69.68 * *$ & 7.74 & 0 & $23.15 * *$ & $-6.11 * *$ & $18.70 * *$ & -0.5 \\
\hline 8 & AKD1X 554-1 & $-50.00 * *$ & $14.73 * *$ & -3.32 & $-58.91 * *$ & $-6.44 * *$ & $-19.38 * *$ & $-22.68 * *$ & -1.24 \\
\hline 9 & AKD1X 636-5 & $-31.43 * *$ & $-6.18 *$ & $-17.94 * *$ & 0 & -0.18 & $-16.98 * *$ & $-15.22 * *$ & 0.4 \\
\hline 10 & AKD1X 2-1-12 & $-16.35 * *$ & $56.40 * *$ & $40.24 * *$ & $31.31 * *$ & $62.15 * *$ & $-3.10 *$ & $53.29 * *$ & 2.68 \\
\hline 11 & $48-1 \times 561-2$ & -4.79 & $55.42 * *$ & $61.54 * *$ & $-58.20 * *$ & $48.21 * *$ & -1.94 & $44.47 * *$ & -3.66 \\
\hline 12 & $48-1 \times 592-2$ & $27.84 * *$ & $15.34 * *$ & $19.05 * *$ & $77.88 * *$ & $15.09 * *$ & $-3.00 *$ & $11.47 * *$ & 0 \\
\hline 13 & 48-1 X 554-1 & $-35.00 * *$ & $41.44 * *$ & $23.34 * *$ & $-73.86 * *$ & $18.80 * *$ & $6.26 * *$ & $25.81 * *$ & -2.1 \\
\hline 14 & $48-1 \times 636-5$ & $-56.36 * *$ & $-40.43 * *$ & $-22.31 * *$ & $-58.20 * *$ & $-31.41 * *$ & 5.19 ** & $-29.42 * *$ & -0.37 \\
\hline 15 & 48-1 X 2-1-12 & $-73.14 * *$ & $-25.24 * *$ & $-18.56 * *$ & $-74.94 * *$ & $-23.82 * *$ & 1.11 & $-23.84 * *$ & 1.52 \\
\hline 16 & Aruna x561-2 & $-29.41 * *$ & $31.78 * *$ & $88.66 * *$ & 0 & $41.00 * *$ & 1.98 & $41.75 * *$ & -1.79 \\
\hline 17 & Aruna x592-2 & $74.83 * *$ & $-4.38 * *$ & -2.34 & 0 & $-12.63 * *$ & $-5.00 * *$ & $-17.39 * *$ & 0.54 \\
\hline 18 & Aruna x554-1 & 1.48 & $6.29 * *$ & $9.80 * *$ & $-58.91 * *$ & -0.85 & $-6.10 * *$ & $-7.73 * *$ & -1.17 \\
\hline $\begin{array}{l}19 \\
\end{array}$ & Aruna x636-5 & $-27.27 * *$ & $-21.48 * *$ & $-22.69 * *$ & 0 & $-28.53 * *$ & $-10.29 * *$ & $-36.02 * *$ & -3.49 \\
\hline 20 & Aruna x 2-1-12 & $21.77 * *$ & 2.39 & $45.69 * *$ & $49.05 * *$ & $28.52 * *$ & $-8.33 * *$ & $17.19 * *$ & 1.3 \\
\hline & $\mathrm{SE}(\mathrm{m}) \pm$ & 0.25 & 0.75 & 0.50 & 0.0512 & 1.22 & 0.36 & 0.99 & 0.83 \\
\hline & CD AT $5 \%$ & 0.51 & 1.52 & 1.00 & 0.1037 & 2.48 & 0.73 & 2.00 & 1.69 \\
\hline & CD AT $1 \%$ & 0.68 & 2.03 & 1.34 & 0.1389 & 3.32 & 0.98 & 2.68 & 2.26 \\
\hline
\end{tabular}

Note: * Significant at $5 \%$ level of significance ** Significant at $1 \%$ level of significance

Table.3 Heterobeltiosis (\%) for various characters

\begin{tabular}{|c|c|c|c|c|c|c|c|c|c|}
\hline $\begin{array}{l}\text { Sr. } \\
\text { No. }\end{array}$ & Crosses & \begin{tabular}{|c|} 
Days to $50 \%$ \\
flowering of \\
primary \\
spikes
\end{tabular} & \begin{tabular}{|c|} 
Days to \\
maturity of \\
primary \\
spikes
\end{tabular} & $\begin{array}{c}\text { Number of } \\
\text { nodes up to } \\
\text { primary } \\
\text { spikes }\end{array}$ & $\begin{array}{c}\text { Effective } \\
\text { length of } \\
\text { primary } \\
\text { spikes }\end{array}$ & $\begin{array}{l}\text { Effective } \\
\text { length of } \\
\text { secondary } \\
\text { spikes }\end{array}$ & $\begin{array}{c}\text { Effective } \\
\text { length of } \\
\text { tertiary } \\
\text { spikes }\end{array}$ & $\begin{array}{c}\text { No. of } \\
\text { secondary } \\
\text { spike }\end{array}$ & $\begin{array}{c}\text { Number of } \\
\text { tertiary } \\
\text { spikes }\end{array}$ \\
\hline & & 1 & 2 & 3 & 4 & 5 & 6 & 7 & 8 \\
\hline 1 & AKC-1 X 561-2 & 3.35 & 0.7 & -8.58 & -0.35 & -2.48 & 3.29 & $-30.33 * *$ & $-11.43 *$ \\
\hline 2 & AKC-1 X 592-2 & $12.44 * *$ & -0.7 & 1.49 & $-41.74 * *$ & $-11.30 *$ & $14.27 * *$ & $-14.26 *$ & $-23.40 * *$ \\
\hline 3 & AKC-1 X554-1 & $13.98 * *$ & -0.7 & $24.02 * *$ & $-46.71 * *$ & $-32.15 * *$ & $-71.12 * *$ & -11.04 & $-57.94 * *$ \\
\hline 4 & AKC-1 X636-5 & -2.87 & 1.39 & -4.75 & $-14.30 * *$ & -0.23 & $-68.86 * *$ & $-24.97 * *$ & $-57.94 * *$ \\
\hline 5 & AKC-1 X 2-1-12 & 0.48 & $3.26 *$ & $41.80 * *$ & $-14.21 * *$ & $-13.39 * *$ & $-73.96 * *$ & $-27.59 * *$ & $-62.46 * *$ \\
\hline 6 & AKD1 x561-2 & $6.35 * *$ & -0.46 & $24.53 * *$ & $71.72 * *$ & $124.77 * *$ & $287.15 * *$ & $43.29 * *$ & $130.72 * *$ \\
\hline 7 & AKD1x592-2 & $13.76^{* *}$ & 2.76 & $20.51 *$ & $-56.99 * *$ & $24.58 * *$ & 0 & -9.22 & 0 \\
\hline 8 & AKD1X 554-1 & $15.59 * *$ & $3.69^{*}$ & $46.28 * *$ & -1.68 & $-22.78 * *$ & $-71.12 * *$ & $-62.50 * *$ & $-51.65 * *$ \\
\hline 9 & AKD1X 636-5 & $14.81 * *$ & 2.3 & $27.06 * *$ & 1.78 & $-40.41 * *$ & 0 & $-53.85 * *$ & 0 \\
\hline 10 & AKD1X 2-1-12 & $-12.70 * *$ & 1.4 & $43.53 * *$ & $46.39 * *$ & $50.27 * *$ & $-13.77 * *$ & $-48.28 * *$ & $-28.27 * *$ \\
\hline 11 & $48-1 \times 561-2$ & $-9.46 * *$ & $-3.79 *$ & -7.99 & $41.86 * *$ & $51.40 * *$ & $-73.26 * *$ & $34.68 * *$ & $-58.79 * *$ \\
\hline 12 & $48-1 \times 592-2$ & $-11.97 * *$ & $4.90 * *$ & 10.58 & $-14.77 * *$ & 6.47 & $8.66 * *$ & 10.82 & -4.92 \\
\hline 13 & $48-1$ X 554-1 & $17.74 * *$ & $-5.35 * *$ & $51.72 * *$ & -10.03 & $15.04 * *$ & $-73.26 * *$ & 0.62 & $-58.79 * *$ \\
\hline 14 & $48-1 \times 636-5$ & $-17.09 * *$ & -1.11 & -4.11 & -10.03 & $-33.86 * *$ & $-73.26 * *$ & $-60.89 * *$ & $-58.79 * *$ \\
\hline 15 & $48-1 \times 2-1-12$ & $-5.09 * *$ & 0.47 & $47.01 * *$ & $-19.36 * *$ & $-29.05 * *$ & $-73.96 * *$ & $-79.31 * *$ & $-62.46^{* *}$ \\
\hline 16 & Aruna x561-2 & $-4.63 * *$ & 1.82 & 7.94 & $-10.04 *$ & $37.87 * *$ & $-72.75 * *$ & $-57.14 * *$ & $-19.62 *$ \\
\hline \begin{tabular}{|l|}
17 \\
\end{tabular} & Aruna x592-2 & $-4.63 * *$ & 0.45 & $16.84 *$ & $-48.90 * *$ & $-28.29 * *$ & $-8.27 * *$ & $18.72 *$ & $46.41 * *$ \\
\hline 18 & Aruna x554-1 & $24.19 * *$ & 0 & $34.17 * *$ & $-28.56 * *$ & $-9.29 *$ & $-72.75 * *$ & 8.38 & $-51.65 * *$ \\
\hline \begin{tabular}{|l|}
19 \\
\end{tabular} & Aruna x636-5 & $-4.17 *$ & $5.23 * *$ & 2.44 & $-33.54 * *$ & $-34.36 * *$ & $-72.75 * *$ & $-53.85 * *$ & $-19.62 *$ \\
\hline 20 & Aruna $x$ 2-1-12 & $-21.76 * *$ & 2.09 & $34.52 * *$ & $-13.55 * *$ & $24.45 * *$ & $4.13 *$ & $-10.34 *$ & $-25.31 * *$ \\
\hline & $\mathrm{SE}(\mathrm{m}) \pm$ & 1.22 & 2.28 & 1.36 & 1.60 & 0.58 & 0.0431 & 0.19 & 0.0742 \\
\hline & CD AT $5 \%$ & 2.47 & 4.61 & 2.75 & 3.25 & 1.17 & 0.0872 & 0.39 & 0.1502 \\
\hline & CD AT $1 \%$ & 3.30 & 6.18 & 3.68 & 4.35 & 1.57 & 0.1168 & 0.52 & 0.2011 \\
\hline
\end{tabular}

Note: * Significant at $5 \%$ level of significance ** Significant at $1 \%$ level of significance 
Table.3 Contd....

\begin{tabular}{|c|c|c|c|c|c|c|c|c|c|}
\hline $\begin{array}{l}\text { Sr. } \\
\text { No. }\end{array}$ & Crosses & $\begin{array}{c}\text { Total } \\
\text { Number of } \\
\text { spikes }\end{array}$ & $\begin{array}{c}\text { Number of } \\
\text { capsules on } \\
\text { primary spikes }\end{array}$ & $\begin{array}{c}\begin{array}{c}\text { Number of } \\
\text { capsules on } \\
\text { secondary spikes }\end{array} \\
\end{array}$ & $\begin{array}{c}\text { Number of } \\
\text { capsules on } \\
\text { tertiary spikes }\end{array}$ & $\begin{array}{c}\text { Total number } \\
\text { of capsule per } \\
\text { plant }\end{array}$ & $\begin{array}{c}100 \text { seed } \\
\text { weight }\end{array}$ & $\begin{array}{l}\text { Seed yield } \\
\text { per plant }\end{array}$ & $\begin{array}{c}\text { Oil } \\
\text { content }\end{array}$ \\
\hline & & 9 & 10 & 11 & 12 & 13 & 14 & 15 & 16 \\
\hline 1 & AKC-1 X 561-2 & $-24.15 * *$ & $25.86 * *$ & $-18.70 * *$ & -4.32 & $11.40 * *$ & $-6.15 * *$ & $4.00 *$ & -0.99 \\
\hline 2 & AKC-1 X 592-2 & $-25.03 * *$ & $21.57 * *$ & -4.07 & 3.37 & $-33.25 * *$ & $-12.17 * *$ & $-42.89 * *$ & -0.46 \\
\hline 3 & AKC-1 X554-1 & $-41.57 * *$ & $-18.70 * *$ & $-39.41 * *$ & $-74.14 * *$ & $-52.03 * *$ & -2.38 & $-53.14 * *$ & -3.22 \\
\hline 4 & AKC-1 X636-5 & $-48.29 * *$ & -0.65 & $-13.50 * *$ & $-72.36 * *$ & $-33.17 * *$ & $-4.51 * *$ & $-37.04 * *$ & $-4.81 *$ \\
\hline 5 & AKC-1 X 2-1-12 & $-49.38 * *$ & $-13.97 * *$ & $-18.56 * *$ & $-76.16 * *$ & $-34.31 * *$ & -1 & $-35.46 * *$ & $5.98 *$ \\
\hline 6 & AKD1 x561-2 & $95.30 * *$ & $71.61 * *$ & $106.39 * *$ & $314.60 * *$ & $97.34 * *$ & $-15.18 * *$ & $114.60 * *$ & $-5.30 *$ \\
\hline 7 & AKD1x592-2 & -6.5 & $36.88 * *$ & 0.28 & 0 & 1.98 & $-15.69 * *$ & $8.25 * *$ & -0.79 \\
\hline 8 & AKD1X 554-1 & $-62.50 * *$ & -1.83 & $-12.28 * *$ & $-74.14 * *$ & $-22.35 * *$ & $-30.26 * *$ & $-26.44 * *$ & -2.56 \\
\hline 9 & AKD1X 636-5 & $-36.84 * *$ & $-21.97 * *$ & $-30.66 * *$ & 0 & $-19.83 * *$ & $-24.51 * *$ & $-26.24 * *$ & -1.58 \\
\hline 10 & AKD1X 2-1-12 & $-45.63 * *$ & $49.04 * *$ & $31.92 * *$ & $-18.69 * *$ & $45.30 * *$ & $-11.79 * *$ & $26.33 * *$ & 1.55 \\
\hline 11 & $48-1 \times 561-2$ & $-25.94 * *$ & $47.16 * *$ & $34.38 * *$ & $-73.58 * *$ & $32.37 * *$ & $-7.91 * *$ & $22.12 * *$ & $-5.43 *$ \\
\hline 12 & $48-1 \times 592-2$ & 0 & $7.16 * *$ & 1.04 & $12.44 * *$ & $10.31 * *$ & $-5.72 * *$ & 3.99 & -0.9 \\
\hline 13 & 48-1 X 554-1 & $-38.61 * *$ & $23.06 * *$ & $22.96 * *$ & $-74.14 * *$ & $13.57 * *$ & -0.85 & $12.60 * *$ & -3.99 \\
\hline 14 & $48-1 \times 636-5$ & $-66.67 * *$ & $-46.58 * *$ & $-28.05 * *$ & $-73.58 * *$ & $-31.66 * *$ & $3.65 *$ & $-30.70 * *$ & -2.93 \\
\hline 15 & $48-1 \times 2-1-12$ & $-77.50 * *$ & $-44.79 * *$ & $-30.22 * *$ & $-76.16 * *$ & $-43.75 * *$ & -0.24 & $-44.32 * *$ & 1.03 \\
\hline 16 & Aruna x561-2 & $-40.00 * *$ & $10.79 * *$ & $58.92 * *$ & 0 & $20.09 * *$ & $-5.44 * *$ & $13.42 * *$ & -2.36 \\
\hline 17 & Aruna x592-2 & $47.64 * *$ & $-20.91 * *$ & $-16.03 * *$ & 0 & $-20.48 * *$ & $-8.88 * *$ & $-27.61 * *$ & -0.99 \\
\hline 18 & Aruna x554-1 & $-27.06 * *$ & $-16.87 * *$ & $8.45 *$ & $-74.14 * *$ & $-9.97 * *$ & $-13.49 * *$ & $-22.19 * *$ & -1.66 \\
\hline 19 & Aruna x636-5 & $-36.84 * *$ & $-36.99 * *$ & $-29.40 * *$ & 0 & $-32.54 * *$ & $-12.78 * *$ & $-41.22 * *$ & -3.65 \\
\hline 20 & Aruna x 2-1-12 & $-23.13 * *$ & $-30.19 * *$ & $26.47 * *$ & $-7.71 * *$ & $-8.33 * *$ & $-10.77 * *$ & $-17.86 * *$ & -1.6 \\
\hline & $\mathrm{SE}(\mathrm{m}) \pm$ & 0.29 & 0.87 & 0.57 & 0.0592 & 1.41 & 0.42 & 1.14 & 0.96 \\
\hline & CD AT $5 \%$ & 0.59 & 1.75 & 1.15 & & 2.86 & 0.85 & 2.31 & 1.95 \\
\hline & CD AT $1 \%$ & 0.79 & 2.35 & 1.55 & 0.1604 & 3.83 & 1.13 & 3.1 & 2.61 \\
\hline
\end{tabular}

Note: * Significant at 5\% level of significance ** Significant at $1 \%$ level of significance

Table.4a Percentage of useful heterosis estimated over the check AKC-1

\begin{tabular}{|c|c|c|c|c|c|c|c|c|c|}
\hline $\begin{array}{l}\text { Sr. } \\
\text { No. }\end{array}$ & Crosses & \begin{tabular}{|c|} 
Days to $50 \%$ \\
flowering of \\
primary spikes
\end{tabular} & $\begin{array}{c}\text { Days to } \\
\text { maturity of } \\
\text { primary } \\
\text { spikes } \\
\end{array}$ & $\begin{array}{c}\text { Number of } \\
\text { nodes up to } \\
\text { primary } \\
\text { spikes } \\
\end{array}$ & $\begin{array}{c}\text { Effective } \\
\text { length of } \\
\text { primary } \\
\text { spikes } \\
\end{array}$ & $\begin{array}{c}\text { Effective } \\
\text { length of } \\
\text { secondary } \\
\text { spikes } \\
\end{array}$ & $\begin{array}{c}\text { Effective } \\
\text { length of } \\
\text { tertiary } \\
\text { spikes } \\
\end{array}$ & $\begin{array}{c}\text { Number of } \\
\text { secondary } \\
\text { spike }\end{array}$ & $\begin{array}{c}\text { Number of } \\
\text { tertiary } \\
\text { spikes }\end{array}$ \\
\hline & & 1 & 2 & 3 & 4 & 5 & 6 & 7 & 8 \\
\hline 1 & AKC-1 X 561-2 & 3.35 & 0.7 & $-12.96 *$ & -0.35 & -2.48 & 3.37 & $-30.33 * *$ & $-11.43 *$ \\
\hline 2 & AKC-1 X 592-2 & $12.44 * *$ & -0.7 & $-13.52 *$ & $-39.29 * *$ & $-11.30 *$ & $14.22 * *$ & $-14.26 *$ & $-23.40 * *$ \\
\hline 3 & AKC-1 X554-1 & 1.44 & -0.7 & 10.42 & $-46.71 * *$ & $-32.15 * *$ & $-68.77 * *$ & -11.04 & $-57.94 * *$ \\
\hline 4 & AKC-1 X636-5 & -2.87 & 1.39 & $-12.46 *$ & $-14.30 * *$ & 8.57 & $-68.77 * *$ & $-24.97 * *$ & $-57.94 * *$ \\
\hline 5 & AKC-1 X 2-1-12 & 0.48 & 3.02 & 9.93 & $-14.21 * *$ & $-13.39 * *$ & $-68.77 * *$ & $12.54 *$ & $-57.94 * *$ \\
\hline 6 & AKD1 x561-2 & $-3.83 *$ & 0.23 & 7.04 & $23.74 * *$ & $44.97 * *$ & $20.53 * *$ & 7.5 & -2.97 \\
\hline 7 & AKD1x592-2 & 2.87 & $3.48 *$ & 10.04 & $-55.18 * *$ & $-17.89 * *$ & $-68.77 * *$ & $-30.33 * *$ & $-57.94 * *$ \\
\hline 8 & AKD1X 554-1 & 2.87 & $4.41 * *$ & 5.65 & $-18.89 * *$ & $-29.44 * *$ & $-68.77 * *$ & $-67.85 * *$ & $-57.94 * *$ \\
\hline 9 & AKD1X 636-5 & $3.83 *$ & 3.02 & 5.15 & $-16.13 * *$ & $-35.16 * *$ & $-68.77 * *$ & $-67.85 * *$ & $-57.94 * *$ \\
\hline 10 & AKD1X 2-1-12 & $-21.05 * *$ & 1.16 & 8.83 & $-22.37 * *$ & -3.08 & 3.08 & $-19.61 * *$ & $-19.64 * *$ \\
\hline 11 & $48-1 \times 561-2$ & $-3.83 *$ & 0.23 & $-13.17 *$ & $21.33 * *$ & $32.60 * *$ & $-68.77 * *$ & 10.72 & $-57.94 * *$ \\
\hline 12 & $48-1 \times 592-2$ & -1.44 & $9.28 * *$ & 5.77 & $-11.20 *$ & -6.76 & $26.54 * *$ & -8.9 & -2.97 \\
\hline 13 & $48-1$ X 554-1 & $4.78 * *$ & -1.39 & 9.58 & $-23.05 * *$ & 5.12 & $-68.77 * *$ & $-13.72 *$ & $-57.94 * *$ \\
\hline 14 & $48-1 \times 636-5$ & $-7.18 * *$ & 3.02 & $-11.87 *$ & $-23.05 * *$ & $-28.02 * *$ & $-68.77 * *$ & $-67.85 * *$ & $-57.94 * *$ \\
\hline 15 & $48-1 \times 2-1-12$ & -1.91 & 0.23 & 6.62 & $-31.03 * *$ & $-37.86 * *$ & $-68.77 * *$ & $-67.85 * *$ & $-57.94 * *$ \\
\hline 16 & Aruna x561-2 & -1.44 & $3.94 *$ & 10.35 & 3.88 & $34.51 * *$ & $-68.77 * *$ & $-67.85 * *$ & $-57.94 * *$ \\
\hline 17 & Aruna x592-2 & -1.44 & 2.55 & 2.96 & $-40.99 * *$ & $-30.04 * *$ & $4.84 *$ & -8.9 & $-23.40 * *$ \\
\hline 18 & Aruna x554-1 & 10.53 ** & 2.09 & 3.10 & $-17.51 * *$ & $-11.50 *$ & $-68.77 * *$ & -7.07 & $-57.94 * *$ \\
\hline 19 & Aruna x636-5 & -0.96 & $7.42 * *$ & $-14.92 *$ & $-23.26 * *$ & $-28.57 * *$ & $-68.77 * *$ & $-67.85 * *$ & $-57.94 * *$ \\
\hline 20 & Aruna x 2-1-12 & $-19.14 * *$ & 1.86 & $-14.55 *$ & -0.18 & $21.42 * *$ & $24.49 * *$ & $39.34 * *$ & $-16.33 * *$ \\
\hline & $\mathrm{SE}(\mathrm{m}) \pm$ & 1.22 & 2.28 & 1.36 & 1.60 & 0.58 & 0.0431 & 0.19 & 0.0742 \\
\hline & CD AT $5 \%$ & 2.47 & 4.61 & 2.75 & 3.25 & 1.17 & 0.0872 & 0.39 & 0.1502 \\
\hline & CD AT $1 \%$ & 3.30 & 6.18 & 3.68 & 4.35 & 1.57 & 0.1168 & 0.52 & 0.2011 \\
\hline
\end{tabular}

Note: * Significant at 5\% level of significance ** Significant at $1 \%$ level of significance 
Table.4a Contd....

\begin{tabular}{|c|c|c|c|c|c|c|c|c|c|}
\hline $\begin{array}{l}\text { Sr. } \\
\text { No. }\end{array}$ & Crosses & $\begin{array}{c}\text { Total Number } \\
\text { of spikes }\end{array}$ & $\begin{array}{c}\text { Number of } \\
\text { capsules on } \\
\text { primary spikes }\end{array}$ & \begin{tabular}{|c} 
Number of \\
capsules on \\
secondary \\
spikes
\end{tabular} & \begin{tabular}{|c} 
Number of \\
capsules on \\
tertiary \\
spikes
\end{tabular} & $\begin{array}{c}\text { Total } \\
\text { number of } \\
\text { capsule per } \\
\text { plant } \\
\end{array}$ & $\begin{array}{c}100 \text { seed } \\
\text { weight }(\mathrm{g})\end{array}$ & $\begin{array}{c}\text { Seed yield } \\
\text { per plant } \\
\text { (g) }\end{array}$ & $\begin{array}{c}\text { Oil } \\
\text { content } \\
(\%)\end{array}$ \\
\hline & & 9 & 10 & 11 & 12 & 13 & 14 & 15 & 16 \\
\hline 1 & AKC-1 X 561-2 & $-24.15 * *$ & $25.86 * *$ & $-18.70 * *$ & -4.32 & $11.40 * *$ & $-6.15 * *$ & $4.00 *$ & 0.79 \\
\hline 2 & AKC-1 X 592-2 & $-25.03 * *$ & $21.57 * *$ & -4.07 & 3.37 & $-33.25 * *$ & $-12.17 * *$ & $-42.89 * *$ & -0.46 \\
\hline 3 & AKC-1 X554-1 & $-41.57 * *$ & $-18.70 * *$ & $-39.41 * *$ & $-72.36 * *$ & $-52.03 * *$ & -2.38 & $-53.14 * *$ & -1.29 \\
\hline 4 & AKC-1 X636-5 & $-48.29 * *$ & -0.65 & $-13.50 * *$ & $-72.36 * *$ & $-33.17 * *$ & $-4.39 * *$ & $-37.04 * *$ & -1.62 \\
\hline 5 & AKC-1 X 2-1-12 & $-30.20 * *$ & $-13.97 * *$ & $-18.56 * *$ & $-72.36 * *$ & $-34.31 * *$ & -0.63 & $-35.46 * *$ & $5.98 *$ \\
\hline 6 & AKD1 x561-2 & 0.98 & $44.35 * *$ & $28.07 * *$ & $14.58 * *$ & $37.17 * *$ & $3.76 *$ & $39.66 * *$ & -3.6 \\
\hline 7 & AKD1x592-2 & $-50.88 * *$ & $10.57 * *$ & $-37.77 * *$ & $-72.36 * *$ & $-17.33 * *$ & 3.14 & $-14.49 * *$ & -0.93 \\
\hline 8 & AKD1X 554-1 & $-68.98 * *$ & $-31.62 * *$ & $-33.18 * *$ & $-72.36 * *$ & $-37.40 * *$ & $-14.68 * *$ & $-46.97 * *$ & -0.61 \\
\hline 9 & AKD1X 636-5 & $-68.98 * *$ & $-41.72 * *$ & $-37.65 * *$ & $-72.36 * *$ & $-29.65 * *$ & $-7.65 * *$ & $-35.14 * *$ & 1.72 \\
\hline 10 & AKD1X 2-1-12 & $-25.03 * *$ & $-26.15 * *$ & $-18.14 * *$ & $-5.74 *$ & $-22.70 * *$ & $7.90 * *$ & $-17.78 * *$ & 0.82 \\
\hline 11 & $48-1 \times 561-2$ & $-31.08 * *$ & $38.52 * *$ & 2.99 & $-72.36 * *$ & $17.02 * *$ & $-5.02 * *$ & $11.43 * *$ & -3.74 \\
\hline 12 & $48-1 \times 592-2$ & -6.93 & 0.86 & $-22.56 * *$ & $17.61 * *$ & -2.48 & -2.76 & $-5.11 * *$ & -1.04 \\
\hline 13 & $48-1$ X 554-1 & $-42.86 * *$ & $15.82 * *$ & -5.76 & $-72.36 * *$ & 0.4 & 2.26 & 2.75 & -2.08 \\
\hline 14 & $48-1 \times 636-5$ & $-68.98 * *$ & $-49.72 * *$ & $-35.30 * *$ & $-72.36 * *$ & $-39.59 * *$ & $6.90 * *$ & $-36.77 * *$ & 0.33 \\
\hline 15 & $48-1 \times 2-1-12$ & $-68.98 * *$ & $-48.03 * *$ & $-46.52 * *$ & $-72.36 * *$ & $-50.28 * *$ & 2.89 & $-49.19 * *$ & -0.94 \\
\hline 16 & Aruna x561-2 & $-68.98 * *$ & $36.78 * *$ & $18.07 * *$ & $-72.36 * *$ & $18.67 * *$ & 0.25 & $19.07 * *$ & 0.57 \\
\hline 17 & Aruna x592-2 & $-22.44 * *$ & -2.35 & $-37.62 * *$ & $-72.36 * *$ & $-21.42 * *$ & $-3.39 *$ & $-24.01 * *$ & 1.98 \\
\hline 18 & Aruna x 554-1 & $-39.66 * *$ & 2.63 & $-17.39 * *$ & $-72.36 * *$ & $-11.04 * *$ & $-8.28 * *$ & $-18.31 * *$ & 1.3 \\
\hline 19 & Aruna x636-5 & $-68.98 * *$ & $-22.21 * *$ & $-36.52 * *$ & $-72.36 * *$ & $-33.33 * *$ & $-7.53 * *$ & $-38.29 * *$ & -0.42 \\
\hline 20 & Aruna x 2-1-12 & 6.00 & $-13.81 * *$ & -6.03 & $7.00 * *$ & $-9.42 * *$ & $-5.40 * *$ & $-13.77 * *$ & 1.35 \\
\hline & $\mathrm{SE}(\mathrm{m}) \pm$ & 0.29 & 0.87 & 0.57 & 0.0592 & 1.41 & 0.42 & 1.14 & 0.96 \\
\hline & CD AT $5 \%$ & 0.59 & 1.75 & 1.16 & 0.1198 & 2.86 & 0.85 & 2.31 & 1.95 \\
\hline & CD AT $1 \%$ & 0.79 & 2.35 & 1.55 & 0.1604 & 3.83 & 1.13 & 3.10 & 2.61 \\
\hline
\end{tabular}

Note: * Significant at $5 \%$ level of significance ** Significant at $1 \%$ level of significance

Table.4b Percentage of useful heterosis estimated over the check 48-1

\begin{tabular}{|c|c|c|c|c|c|c|c|c|c|}
\hline $\begin{array}{c}\text { Sr. } \\
\text { No. }\end{array}$ & Crosses & \begin{tabular}{|c|} 
Days to $50 \%$ \\
flowering of \\
primary spikes
\end{tabular} & $\begin{array}{c}\text { Days to } \\
\text { maturity of } \\
\text { primary } \\
\text { spikes }\end{array}$ & \begin{tabular}{|c|}
$\begin{array}{c}\text { Number of } \\
\text { nodes up to } \\
\text { primary } \\
\text { spikes }\end{array}$ \\
\end{tabular} & $\begin{array}{c}\text { Effective } \\
\text { length of } \\
\text { primary } \\
\text { spikes }\end{array}$ & $\begin{array}{l}\text { Effective } \\
\text { length of } \\
\text { secondary } \\
\text { spikes }\end{array}$ & $\begin{array}{l}\text { Effective } \\
\text { length of } \\
\text { tertiary } \\
\text { spikes }\end{array}$ & $\begin{array}{c}\text { Number } \\
\text { of } \\
\text { Secondary } \\
\text { spike }\end{array}$ & $\begin{array}{c}\text { Number of } \\
\text { tertiary } \\
\text { spike }\end{array}$ \\
\hline & & 1 & 2 & \begin{tabular}{|l|}
3 \\
\end{tabular} & 4 & 5 & 6 & 7 & 8 \\
\hline 1 & AKC-1 X 561-2 & $-7.69 * *$ & $-3.34 *$ & 7.76 & $16.51 * *$ & $11.35 *$ & $-11.21 * *$ & $-15.25 *$ & $-13.21 * *$ \\
\hline 2 & AKC-1 X 592-2 & 0.43 & $-4.68 * *$ & 8.36 & $-29.02 * *$ & 1.28 & -1.89 & 4.3 & $-24.94 * *$ \\
\hline 3 & AKC-1 X554-1 & $-9.40 * *$ & $-4.68 * *$ & 5.07 & $-37.69 * *$ & $-22.52 * *$ & $-73.26 * *$ & 8.21 & $-58.79 * *$ \\
\hline 4 & AKC-1 X636-5 & $-13.25 * *$ & -2.67 & 7.24 & 0.21 & $23.97 * *$ & $-73.26 * *$ & -8.74 & $-58.79 * *$ \\
\hline 5 & AKC-1 X 2-1-12 & $-10.26 * *$ & -1.11 & 4.55 & 0.3 & -1.11 & $-73.26 * *$ & $36.90 * *$ & $-58.79 * *$ \\
\hline 6 & AKD1 x561-2 & $-14.10 * *$ & $-3.79 *$ & 1.49 & $44.68 * *$ & $65.53 * *$ & $3.52 *$ & $30.77 * *$ & -4.92 \\
\hline 7 & AKD1x592-2 & $-8.12 * *$ & -0.67 & 4.67 & $-47.60 * *$ & -6.24 & $-73.26 * *$ & $-15.25 *$ & $-58.79 * *$ \\
\hline 8 & AKD1X 554-1 & $-8.12 * *$ & 0.22 & 11.96 & -5.17 & $-19.43 * *$ & $-73.26 * *$ & $-60.89 * *$ & $-58.79 * *$ \\
\hline 9 & AKD1X 636-5 & $-7.26 * *$ & -1.11 & 0.51 & -1.93 & $-25.96 * *$ & $-73.26 * *$ & $-60.89 * *$ & $-58.79 * *$ \\
\hline 10 & AKD1X 2-1-12 & $-29.48 * *$ & -2.9 & 3.39 & -9.23 & $10.67 *$ & $-11.47 * *$ & -2.22 & $-21.26 * *$ \\
\hline 11 & $48-1 \times 561-2$ & $-14.10 * *$ & $-3.79 *$ & 7.99 & $41.86 * *$ & $51.40 * *$ & $-73.26 * *$ & $34.68 * *$ & $-58.79 * *$ \\
\hline 12 & $48-1 \times 592-2$ & $-11.97 * *$ & $4.90 * *$ & 0.15 & 3.83 & 6.47 & $8.66 * *$ & 10.82 & -4.92 \\
\hline 13 & 48-1 X 554-1 & $-6.41 * *$ & $-5.35 * *$ & $16.12 *$ & -10.03 & $20.03 * *$ & $-73.26 * *$ & 4.95 & $-58.79 * *$ \\
\hline 14 & $48-1$ X 636-5 & $-17.09 * *$ & -1.11 & 6.61 & -10.03 & $-17.82 * *$ & $-73.26 * *$ & $-60.89 * *$ & $-58.79 * *$ \\
\hline 15 & $48-1$ X 2-1-12 & $-12.39 * *$ & $-3.79 *$ & 1.04 & $-19.36 * *$ & $-29.05 * *$ & $-73.26 * *$ & $-60.89 * *$ & $-58.79 * *$ \\
\hline 16 & Aruna x561-2 & $-11.97 * *$ & -0.22 & 5.00 & $21.46 * *$ & $53.59 * *$ & $-73.26 * *$ & $-60.89 * *$ & $-58.79 * *$ \\
\hline 17 & Aruna x592-2 & $-11.97 * *$ & -1.56 & 2.84 & $-31.00 * *$ & $-20.11 * *$ & $-10.01 * *$ & 10.82 & $-24.94 * *$ \\
\hline 18 & Aruna x554-1 & -1.28 & -2 & 2.69 & -3.55 & 1.05 & $-73.26 * *$ & 13.04 & $-58.79 * *$ \\
\hline 19 & Aruna x636-5 & $-11.54 * *$ & $3.12 *$ & 9.84 & -10.27 & $-18.44 * *$ & $-73.26 * *$ & $-60.89 * *$ & $-58.79 * *$ \\
\hline 20 & Aruna x 2-1-12 & $-28.20 * *$ & -2.23 & 9.45 & $16.72 * *$ & $38.64 * *$ & $6.92 * *$ & $69.49 * *$ & $-18.01 * *$ \\
\hline & $\mathrm{SE}(\mathrm{m}) \pm$ & 1.22 & 2.28 & 1.36 & 1.60 & 0.58 & 0.0431 & 0.19 & 0.0742 \\
\hline & CD AT $5 \%$ & 2.47 & 4.61 & 2.75 & 3.25 & 1.17 & 0.0872 & 0.39 & 0.1502 \\
\hline & CD AT $1 \%$ & 3.30 & 6.18 & 3.68 & 4.35 & 1.57 & 0.1168 & 0.52 & 0.2011 \\
\hline
\end{tabular}

Note: * Significant at $5 \%$ level of significance ** Significant at $1 \%$ level of significance 
Table.4b Contd...

\begin{tabular}{|c|c|c|c|c|c|c|c|c|c|}
\hline $\begin{array}{l}\text { Sr. } \\
\text { No. }\end{array}$ & Crosses & $\begin{array}{c}\text { Total } \\
\text { Number of } \\
\text { spikes }\end{array}$ & $\begin{array}{c}\text { Number of } \\
\text { capsules on } \\
\text { primary spikes }\end{array}$ & $\begin{array}{c}\text { Number of } \\
\text { capsules on } \\
\text { secondary spikes }\end{array}$ & \begin{tabular}{|c|} 
Number of \\
capsules on \\
tertiary spikes
\end{tabular} & $\begin{array}{c}\text { Total number } \\
\text { of capsule per } \\
\text { plant }\end{array}$ & $\begin{array}{c}100 \text { seed } \\
\text { weight }(g)\end{array}$ & \begin{tabular}{|c} 
Seed \\
yield per \\
plant (g)
\end{tabular} & $\begin{array}{c}\text { Oil } \\
\text { content } \\
(\%)\end{array}$ \\
\hline & & 9 & 10 & 11 & 12 & 13 & 14 & 15 & 16 \\
\hline 1 & AKC-1 X 561-2 & $-18.50 * *$ & $33.72 * *$ & 6.08 & $-8.52 * *$ & $26.01 * *$ & *** & $13.98 * *$ & 2.79 \\
\hline 2 & AKC-1 X 592-2 & $-19.44 * *$ & $29.16 * *$ & $25.16 * *$ & -1.18 & $-24.49 * *$ & $-14.84 * *$ & $-37.41 * *$ & 1.52 \\
\hline 3 & AKC-1 X554-1 & $-37.22 * *$ & $-13.63 * *$ & $-20.94 * *$ & $-73.58 * *$ & $-45.73 * *$ & $-5.35 * *$ & $-48.65 * *$ & 0.67 \\
\hline 4 & AKC-1 X636-5 & $-44.44 * *$ & $5.55 * *$ & $12.87 * *$ & $-73.58 * *$ & $-24.41 * *$ & $-7.30 * *$ & $-31.00 * *$ & 0.34 \\
\hline 5 & AKC-1 X 2-1-12 & $-25.00 * *$ & $-8.60 * *$ & 6.26 & $-73.58 * *$ & $-25.69 * *$ & $-3.65 *$ & $-29.27 * *$ & 8.09 ** \\
\hline 6 & AKD1 x561-2 & 8.5 & $53.37 * *$ & $67.10 * *$ & $9.55 * *$ & $55.16 * *$ & 0.61 & $53.05 * *$ & -1.68 \\
\hline 7 & AKD1x592-2 & $-47.22 * *$ & $17.47 * *$ & $-18.81 * *$ & $-73.58 * *$ & $-6.48 * *$ & 0 & $-6.29 * *$ & 1.04 \\
\hline 8 & AKD1X 554-1 & $-66.67 * *$ & $-27.35 * *$ & $-12.82 * *$ & $-73.58 * *$ & $-29.18 * *$ & $-17.27 * *$ & $-41.89 * *$ & 1.36 \\
\hline 9 & AKD1X 636-5 & $-66.67 * *$ & $-38.08 * *$ & $-18.65 * *$ & $-73.58 * *$ & $-20.43 * *$ & $-10.46 * *$ & $-28.92 * *$ & 3.74 \\
\hline 10 & AKD1X 2-1-12 & $-19.44 * *$ & $-21.54 * *$ & 6.81 & $-9.88 * *$ & $-12.56 * *$ & $4.62 * *$ & $-9.90 * *$ & 2.82 \\
\hline 11 & $48-1 \times 561-2$ & $-25.94 * *$ & 47.16 ** & $34.38 * *$ & $-73.58 * *$ & $32.37 * *$ & $-7.91 * *$ & $22.12 * *$ & -1.82 \\
\hline 12 & $48-1 \times 592-2$ & 0 & $7.16 * *$ & 1.04 & $12.44 * *$ & $10.31 * *$ & $-5.72 * *$ & 3.99 & 0.93 \\
\hline 13 & $48-1$ X 554-1 & $-38.61 * *$ & $23.06 * *$ & $22.96 * *$ & $-73.58 * *$ & $13.57 * *$ & -0.85 & $12.60 * *$ & -0.13 \\
\hline 14 & $48-1$ X 636-5 & $-66.67 * *$ & $-46.58 * *$ & $-15.59 * *$ & $-73.58 * *$ & $-31.66 * *$ & $3.65 *$ & $-30.70 * *$ & 2.32 \\
\hline 15 & $48-1 \times 2-1-12$ & $-66.67 * *$ & $-44.79 * *$ & $-30.22 * *$ & $-73.58 * *$ & $-43.75 * *$ & -0.24 & $-44.32 * *$ & 1.03 \\
\hline 16 & Aruna x561-2 & $-66.67 * *$ & $45.32 * *$ & $54.05 * *$ & $-73.58 * *$ & $34.23 * *$ & -2.8 & $30.48 * *$ & 2.57 \\
\hline 17 & Aruna x592-2 & $-16.67 * *$ & $3.74 *$ & $-18.61 * *$ & $-73.58 * *$ & $-11.12 * *$ & $-6.33 * *$ & $-16.72 * *$ & 4.01 \\
\hline 18 & Aruna x554-1 & $-35.17 * *$ & $9.04 * *$ & 7.78 & $-73.58 * *$ & 0.63 & $-11.07 * *$ & $-10.48 * *$ & 3.31 \\
\hline 19 & Aruna x636-5 & $-66.67 * *$ & $-17.35 * *$ & $-17.18 * *$ & $-73.58 * *$ & $-24.59 * *$ & $-10.34 * *$ & $-32.37 * *$ & 1.56 \\
\hline 20 & Aruna x 2-1-12 & $13.89 * *$ & $-8.43 * *$ & $22.60 * *$ & 2.3 & 2.47 & $-8.27 * *$ & $-5.50 * *$ & 3.37 \\
\hline & $\mathrm{SE}(\mathrm{m}) \pm$ & 0.29 & 0.87 & 0.57 & 0.0592 & 1.41 & 0.42 & 1.14 & 0.96 \\
\hline & CD AT $5 \%$ & 0.59 & 1.75 & 1.16 & 0.1198 & 2.86 & 0.85 & 2.31 & 1.95 \\
\hline & CD AT $1 \%$ & 0.79 & 2.35 & 1.55 & 0.1604 & 3.83 & 1.13 & 3.10 & 2.61 \\
\hline
\end{tabular}

Note: * Significant at $5 \%$ level of significance ** Significant at $1 \%$ level of significance

This study thus, substantiates the findings of Kaul and Prasad (1983), Mehta et al., (1991) and Joshi (1993) Patel et al., (2013).

The yield superiority of a cross over best cultivated varieties is essential from commercial cultivation point of view. The crosses showing maximum useful heterosis over the check AKC-1 and 48-1 for different characters in desired direction are given in Table 4(a) and 4(b) respectively. The highest useful heterosis over the check AKC-1 in desirable direction was recorded by the cross AKD1 X 561-2 for maximum number of characters viz., effective length of primary spikes (23.74\%), effective length of secondary spikes (44.97\%), effective length of tertiary spikes $(20.53 \%)$, number of capsules on primary spikes $(44.35 \%)$, number of capsule on secondary spikes $(28.07 \%)$, number of capsule on tertiary spikes $(14.58 \%)$, number of capsule per plant $(31.17 \%)$, seed yield (39.66\%) The cross 48-1 x 636-5 showed highest useful heterosis over the check AKC-1 for 100 seed weight (6.90\%). The cross AKD1 x 2-1-12 displayed maximum useful heterosis over the check AKC-1 for days to $50 \%$ flowering $(-21.05 \%)$ in desired direction. The cross Aruna x 2-1-12 exhibited maximum useful heterosis over the check AKC-1 for number of secondary spikes (39.34\%). The cross AKC1 X 2-1-12 (5.98\%) showed significant useful heterosis over AKC1 for oil content and Aruna x 636-5 ($14.92 \%$ ) showed the negative useful heterosis for number of nodes upto primary spikes.

The highest useful heterosis over check 48-1 in desirable direction was recorded by the cross AKD1 x 561-2 for the characters viz., effective length of primary spikes $(44.68 \%)$, effective length of secondary spikes $(65.53 \%)$ number of capsules on primary spikes $(53.37 \%)$, number of secondary spikes $(67.10 \%)$, number of capsules on tertiary spikes $(9.55 \%)$, total number of capsules per 
plant $(55.16 \%)$ and seed yield (53.05\%) The cross Aruna x 2-1-12 showed highest useful heterosis over the check 48-1 for the traits viz., number of secondary spikes (36.90\%). The cross AKD1 X 2-1-12 displayed highest negative useful heterosis for days to $50 \%$ flowering (-29.49) and AKC1 X 2-1-12 for oil content $(8.09 \%)$. High association of heterosis between these characters and seed yield per plant in castor has also been earlier reported by Varisai et al., 1970; Saiyed et al., 1997; Joshi et al., 2001; Chandramohan and Lavanya; 2003; Thakker et al., 2005; Chaudhari, 2006; Patel et al., 2006; Sridhar et al., 2009; Ramesh et al., 2013 and Chaudhari et al.,2014

From the present study, it can be concluded that considerable heterosis, heterobeltiosis and standard heterosis observed for seed yield and other associated characters suggested the presence of large genetic diversity among the parents and also unidirectional distribution of allelic constitution contributing towards desirable heterosis in the present material. Overall, results of heterosis analysis revealed that the cross AKD1 X 561-2 exhibited highest average heterosis (118.05\%), heterobeltiosis $(114.60 \%)$ and standard heterosis over the check AKC-1 (39.66\%) and the check $48-1(53.05 \%)$ for seed yield per plant. Therefore, the potential of this cross should have to be confirmed for its further utilization in improvement of this crop.

\section{References}

Barad, Y.M., Pathak, A.R. and Patel, B.N., 2009. Heterosis for yield and yield attributing traits in castor (Ricinus communis L.). J. Oilseeds Res., 26(2): 102-104

Chandramohan, Y. and C. Lavanya, 2003. Combining ability and heterosis for seed yield and yield components in castor, Ricinus communis L. J. Oilseeds
Res., 20(2): 220-224.

Chaudhari, G. and Patel, B. N., (2014). Heterosis and combining ability analysis for oil yield and its components in castor (Ricinus communis L.). Trends Biosci., 7(22): 3757-3760.

Chaudhari, K. N. 2006. Diallel analysis for seed yield and wilt resistance in castor. Ph. D. (Agri.) Thesis (Unpublished) submitted to Sardar Krushinagar Datiwada agricultural University, Sardarkrushinagar.

Fonseca, S. and Patterson, F.L., 1968. Hybrid vigour in a seven parent's diallel cross in common winter wheat ( $T$. aestivum L.). Crop Sci., 8: 85-88.

Joshi, H. L., 1993. Heterosis and combining ability studies in castor (Ricinus communis L.). Ph.D. Thesis (Unpublished) submitted to Gujarat Agricultural University, Sardarkrushinagar.

Joshi, H.L., Mehta, D.R. and Jadon, B.S. 2001. Heterosis of yield and yield components in castor hybrids. J. Oilseeds Res., 18 (2) : 164-169.

Kaul, S. L. and M. V. R. Prasad, 1983. Genetic basis of yield heterosis in castor. Pre-congress scientific meeting on genetics and improvement of heterotic system, Coimbatore, India. pp. 15-16 [Fide: Pl. Breed. Abstr., 64(7) : 5348].

Mehta, D. R., P. S. Vashi and M. U. Kukadia, 1991. Hybrid vigour in castor. GAU Res. J., 17(1): 16-22.

Meredith, W.R. and Bridge, R.R., 1972. Heterosis and gene action in cotton, $G$. hirsutum L. Crop Sci., 12: 304-310.

Panse, V. G. and P. V. Sukhatme, 1967. Statistical methods for agricultural workers. Fourth edn. ICAR, New Delhi, pp.360.

Patel, A. R., Patel, K.V. and Patel, J.A., 2013. Extent of heterotic effects for seed yield and component characters in castor 
(Ricinus communis L.) under semi rabi condition. Indian J. Agric. Res., 47(4): 368-372

Patel, J.B. and Pathak, H.C., 2006. Heterosis for seed yield per plant and its components in castor (Ricinus communis L.). J. Oilseeds Res., 23(1): 93-95

Ramana, P.V., Lavanya, C. and Ratnasree, P., 2005. Combining ability and heterosis studies under rainfed conditions in castor (Ricinus communis L.). $J$. Oilseeds Res., 65(4): 325-326

Ramesh, M., C. Lavanya, M. Sujatha, A. Sivasankar, J. A. Kumari and H. P. Meena, 2013. Heterosis and combining ability for yield and yield component characters of newly developed castor (Ricinus communis L.) hybrid. The Bioscan, 8(4): 1421-1424.

Saiyed, M.P., Shukla, P.T. and Dhameliya, H.R., 1997. Heterosis for yield and yield components in castor (Ricinus communis L.) over environments. GAU Res. J., 23(1): 22-27.
Sapovadiya, M.H., Dobariya, K.L., Babariya, C.A., Mungra, K.S. and Vavdiya, P. A., 2015. Heterosis for seed yield and its components over environments in castor (Ricinus communis L.). Elec. J. Pl. Breed., 6(4): 1118-1123

Sridhar, V., Dangi, K.S., Reddy, A.V., Sudhakar, R. and Sankar, A.S., 2009. Heterosis for seed yield and yield components in castor (Ricinus communis L.). Int. J. Agric. Bio., 2(1): 64-67.

Thakkar, D. A., B. S. Jadon, K. M. Patel and C. J. Patel, 2005. Heterosis over environments for seed yield and other attributes in castor, Ricinus communis L. J. Oilseeds Res., 22(2): 324-326.

Varisai, M. S., N. Srinivasalu, M. D. Stephen, K. Navakodi, N. M. Ramaswamy and M. R. Sivaram, 1970. Hybrid vigour in castor and general combining ability between certain Egyptian inbreds and TMV-1. Madras Agric. J., 56(5): 244251.

\section{How to cite this article:}

Sumit G. Gajbhe, S.B. Sakhare and Rathi, S.R. 2017. Exploitation of Heterosis for Yield and its Components in Castor (Ricinus communis L.). Int.J.Curr.Microbiol.App.Sci. 6(12): 2843-2852. doi: https://doi.org/10.20546/ijcmas.2017.612.331 